\title{
The 23 November 2003 Total Solar Eclipse in Antarctica
}

\author{
Jay M. Pasachoff \\ Williams College, Williamstown, MA 01267, USA
}

\begin{abstract}
Totality at the eclipse of 23 November 2003 will cross land only on Antarctica. Details of the path and contact information for safe observation are provided.
\end{abstract}

The total solar eclipse of 23 November 2003 will be visible only from Antarctica and the nearby ocean. The path of totality extends from Mirny at 93 degrees E to the Maitri Novolazarevskaya base at 12 degrees E. Totality lasts from 1 minute 54 seconds at Mirny with the Sun at an altitude of 14 degrees; to a maximum of 1 minute 57 seconds at greatest eclipse, halfway in toward Vostok, with the Sun at an altitude of 18 degrees; to 1 minute 20 seconds with the sun 2 degrees above the horizon where the path leaves the coast near Maitri. The rest of Antarctica will have only a partial eclipse, with the Sun's diameter $77 \%$ covered at McMurdo and 65\% covered at the tip near South America. An ice-breaker passenger ship is planning a 28-day voyage and airplanes are being arranged for observation, including single-day overflights leaving from Melbourne, Australia, and from Punta Arenas, Chile. Scientific observations will include electronic imaging of the corona to compare with simultaneous space observations of the Sun. Links to maps and other items of coordination can be found at www.eclipses.info and www.totalsolareclipse.net, the sites of the IAU Program Group on Public Education at the Time of Eclipses and of the IAU Working Group on Eclipses, respectively. The NASA site with maps and other information is at sunearth.gsfc.nasa.gov/TSE2003/TSE2003.html. Special filters must be used to reduce the solar disk to a safe intensity during the partial phases; only during totality can one look safely without filters. 